\title{
An early complication of transobturator tape: Non-infective adductor internus myositis*
}

\author{
Marc A. Lucky ${ }^{\#}$, Paul Irwin, Saleem Bicha \\ Mid Cheshire Hospitals NHS Foundation Trust, Crewe, UK \\ Email: \#mlucky@nhs.net
}

Received 10 April 2012; revised 23 May 2012; accepted 5 June 2012

\begin{abstract}
The transobturator tape (TOT) procedure is generally felt to be a safer surgical alternative to the tension-free vaginal tape procedure for women with stress urinary incontinence. We report a case of adductor internus myositis not associated with infection following the TOT procedure. To our knowledge this is the first case of this type reported in the literature. A 43 year old lady underwent a straightforward elective TOT procedure. There were no intraoperative complications. Immediately following the procedure she complained of pain in her right thigh. MRI confirmed abnormal oedema within the antero-inferior aspect of the right obturator internus muscle consistent with myositis secondary to tape insertion. The tape was removed the following day in theatre, following which her pain resolved. This case highlights a previously unreported complication as a result of the TOT procedure.
\end{abstract}

Keywords: Transobturator Tape; Adductor Internus Myositis; Complication

\section{INTRODUCTION}

The tension-free vaginal tape procedure has been used widely in the past for female stress incontinence. This procedure is associated with complications such as bladder perforation, excessive bleeding, tape erosion, nerve damage and haematoma formation [1]. An alternative is the transobturator tape (TOT) procedure. It is thought to be associated with a lower risk of complications and lower post-operative morbidity [2]. In this minimally invasive procedure specially designed introducers allow the accurate placement of a synthetic tape behind the urethra and through the obturator foramina towards the

\footnotetext{
${ }^{*}$ Conflicts of interest: No conflicts of interest.

Disclosures: None.

Funding: None.

\#Corresponding author.
}

thigh folds in line with the clitoris. The tape is positioned without tension, forming a sling under the junction of the mid and distal urethra. The trajectory of the tape is such that it is coursed away from the obturator, femoral and saphenous nerves and vessels.

However, the TOT procedure is not without risks. As with all synthetic materials the risk of infection, erosion and myositis exists. The published available literature regarding these complications is scarce. De Souza et al. discussed a case of adductor brevis myositis in a 43 year old woman with cellulitis and vaginal discharge who had a visibly eroded mesh [3]. Leanza et al. also published a report of a late complication of abscess and myositis after insertion of a TOT [4]. These are the only cases reported in the literature of myositis of adductor muscles associated with infection as a possible complication following the TOT procedure. We therefore review and describe a unique case of a symptomatic patient suggestive of adductor internus myositis following a TOT procedure, in which there was no evidence of infection. To our knowledge, this is the first case reporting adductor myositis following a TOT procedure without infection in the literature.

\section{CASE PRESENTATION}

A 43 year old lady was admitted for an elective TOT procedure. She had a 5 year history of urinary incontinence which had not responded to anticholinergic medication and pelvic floor physiotherapy. The procedure was performed without any complication; immediate postplacement cystoscopy confirmed that there was no perforation of the bladder. In the recovery room she complained of severe sharp shooting pains in her right thigh upon movement and felt as though she had a "dead” leg. There was no vascular compromise, paraesthesia, swelling, muscular tenderness or calf pain. She was observed overnight and commenced on tramadol.

Her pain persisted overnight, now being located mainly in the antero-medial and mid thigh level. She also had pain on movement and weight bearing. There was no 
vascular compromise or paresthesia. Her white cell count and inflammatory markers remained normal, and there was no evidence of localised cellulitis. She remained afebrile.

A MRI scan showed abnormal oedema within the antero-inferior aspect of the right obturator internus muscle. No haematoma or abscess was seen. A decision was made to return to theatre to remove part of the tape. The lateral end of the tape on the right side was subsequently removed through the original incision, without complication under general anaesthetic. There was no evidence of infection at the time of or following the procedure. The remainder of the tape was left in place. Upon recovery, the patient immediately reported a dramatic cessation of pain. The following day she was mobilizing well and she was discharged 4 days after the original TOT procedure. Her stress urinary incontinence returned a few days later. She has subsequently had a successful colposuspension.

\section{DISCUSSION}

The TOT procedure is a safe and effective treatment option for women suffering with stress incontinence, associated with a very low risk of complications [5]. The procedure can be done under general or local anaesthetic. The patient is placed in exaggerated lithotomy position with thighs in hyperflexion and slight abduction. A Foley's catheter is placed in the urethra. Two small incisions are made in the inferior part of the obturator for amen at the level of the clitoris. A $2-3 \mathrm{~cm}$ incision is made in the midline of the vagina just under the midurethra, allowing the mesh tape to be placed under the urethra in the correct position. Blunt dissection is made in the direction of the ischiopubic rami. A curved tunneler is then used to place the mesh. The distal segment of the tunneler is manipulated to pass through the obturator membrane. The mesh is then passed through the adductor brevis, obturator externus then obturator internus, using a pronating motion. The tunnelers carrying the mesh are brought out through the skin incision in the groin area. The mesh is then placed under the urethra ensuring it is tension free. Excess tape is trimmed and incisions are sutured.

We believe that this case report is the first to highlight a possible complication of non-infective myositis resulting from the TOT procedure. In this case there was no reason to suspect surgical error. The operation was car- ried out by an experienced urologist who had done many of these procedures before and there were no unexpected problems. The symptoms experienced by the patient could possibly be explained by the course of the tape through the various anatomical structures resulting in inflammation or irritation of muscle tissue. This case is useful from an educational point of view in highlighting a potential complication of this otherwise safe procedure. This case also demonstrates that in patients with unilateral symptoms, not associated with infection, it may only be necessary to remove part of the tape thereby still leaving a chance that the patient's stress incontinence may be improved in spite of further intervention.

\section{CONCLUSION}

It is important that should a patient suffer with any neurological type symptoms following a TOT procedure that imaging in the form of CT or MRI should be done urgently to assess further, with a low threshold for removing the entire or part of the tape.

\section{REFERENCES}

[1] Karram, M.M., Segal, J.L., Vassallo, B.J. and Kleenman, S.D. (2003) Complications and untoward effects of the tension-free vaginal tape procedure. Obstetrics and Gynaecology, 101, 929-932. doi:10.1016/S0029-7844(03)00122-4

[2] Barber, M.D., Gustilo-Ashby, A.M., Chen, C.C., Kaplan, P., Paraiso, M.F. and Walters, M.D. (2006) Perioperative complications and adverse events of the MONARC transobturator tape, compared with the tension-free vaginal tape. American Journal of Obstetrics \& Gynecology, 195, 1820-1825. doi:10.1016/j.ajog.2006.07.007

[3] De Souza, R., Shapiro, A. and Westney, O.L. (2007) Adductor brevis myositis following transobturator tape procedure: A case report and review of the literature. International Urogynecology Journal and Pelvic Floor Dysfunction, 18, 817-820. doi:10.1007/s00192-006-0220-9

[4] Leanza, V., Garozzo, V., Accardi, M., Molino, A., Conco, M. and Basile, A. (2008) A late complication of transobturator tape: Abscess and myositis. Minerva Ginecologica, 60, 91-94.

[5] Costa, P. and Grise, P. (2004) Surgical treatment of female stress urinary incontinence with a trans-obturatortape (TOT) Uratape: Short term results of a prospective multicentric study. European Urology, 46, 102-107. doi:10.1016/j.eururo.2004.03.005 